\title{
Amphoteric arsenic in GaN
}

\author{
U. Wahl ${ }^{\mathrm{a}}$ \\ Instituto Tecnológico e Nuclear, Estrada Nacional 10, 2686-953 Sacavém, Portugal, and \\ Centro de Física Nuclear da Universidade de Lisboa, Avenida Professor Gama Pinto 2, 1649-003 Lisboa, Portugal
}

J. G. Correia

Instituto Tecnológico e Nuclear, Estrada Nacional 10, 2686-953 Sacavém, Portugal, and

Centro de Física Nuclear da Universidade de Lisboa, Avenida Professor Gama Pinto 2, 1649-003 Lisboa, Portugal, and CERN-PH, 1211 Geneva 23, Switzerland

\author{
J.P. Araújo \\ Departamento de Física, Universidade do Porto, Rua do Campo Alegre 687, 4169-007 Porto, Portugal \\ E. Rita and J.C. Soares \\ Centro de Física Nuclear da Universidade de Lisboa, Avenida Professor Gama Pinto 2, 1649-003 Lisboa, Portugal \\ The ISOLDE collaboration \\ CERN-PH, 1211 Geneva 23, Switzerland
}

(Received 15 March 2007; accepted 11 April 2007; published online 4 May 2007)

\begin{abstract}
We have determined the lattice location of implanted arsenic in GaN by means of conversion electron emission channeling from radioactive ${ }^{73}$ As. We give direct evidence that As is an amphoteric impurity, thus settling the long-standing question as to whether it prefers cation or anion sites in GaN. The amphoteric character of As and the fact that As ${ }_{\mathrm{Ga}}$ "antisites" are not minority defects provide additional aspects to be taken into account for an explanantion of the so-called "miscibility gap" in ternary GaAs ${ }_{1-x} \mathrm{~N}_{x}$ compounds, which cannot be grown with a single phase for values of $x$ in the range $0.1<x<0.99$. (C) 2007 American Institute of Physics. [DOI: 10.1063/1.2736299]
\end{abstract}

The growth and properties of ternary semiconductors of the nitride family have been under intense investigation ever since nitrides emerged as blue-light emitting devices in the 1990s. The particular interest in the ternary nitrides results from the fact that they allow adjusting the semiconductor band gap to values not available in pure compounds. One of the ternary systems under study is $\mathrm{GaAs}_{1-x} \mathrm{~N}_{x}$, which shows some intriguing properties such as large band gap bowing parameter [1-3]. Unfortunately the growth of $\mathrm{GaAs}_{1-x} \mathrm{~N}_{x}$ compounds encounters significant difficulties, one of the reasons being that GaAs crystallizes in the cubic zinc blende structure while the most stable polytype of GaN is hexagonal wurtzite. However, while, on the As-rich side of the phase diagram, it is possible to incorporate up to $\sim 10-15 \%$ of $\mathrm{N}$ into cubic GaAs [3, 4], on the $\mathrm{N}$-rich side not more than $\sim 1 \%$ of As in GaN have been achieved [5-8]. In the intermediate region, usually the coexistence of hexagonal N-rich GaAsN and cubic As-rich GaNAs phases is observed.

$\mathrm{GaN}$ which is lightly As-doped is also highly interesting due to the fact that it shows intense blue luminescence centered around $2.6 \mathrm{eV}$, as observed already many years ago in Asimplanted GaN by Pankove and Hutchby [9] and Metcalfe et al [10], and subsequently also found in GaN doped with As during growth [5, 11-16]. The chemical nature of the $2.6 \mathrm{eV}$ blue luminescence and the fact that it results from optical centers involving one As atom only was unambiguously proven by means of the radiotracer photoluminescence (PL) work of Stötzler et al [17], who studied the luminescence along the radioactive decay chains ${ }^{71} \mathrm{As} \rightarrow{ }^{71} \mathrm{Ge} \rightarrow{ }^{71} \mathrm{Ga}$ and ${ }^{72} \mathrm{Se} \rightarrow{ }^{72} \mathrm{As} \rightarrow{ }^{72} \mathrm{Ge}$. Following the ion implantation of ${ }^{71} \mathrm{As}$ and ${ }^{72} \mathrm{Se}$ they observed the intensity of the $2.6 \mathrm{eV}$ luminescence to scale with the amount of radioactive ${ }^{71}$ As and ${ }^{72}$ As resulting from radioactive decay.

The amphoteric nature of As in GaN was first proposed by Guido et al [18], who suggested that As could fill up both Ga and $\mathrm{N}$ vacancies and thus reduce yellow band emission and carrier scattering in GaN. Subsequently, the $2.6 \mathrm{eV}$ blue luminescence $[12,14-16,19]$ and a deep level $\sim 0.8 \mathrm{eV}$ below the conduction band have been attributed to $\mathrm{As}_{\mathrm{Ga}}[20,21]$. While there are indications that the $\mathrm{As}_{\mathrm{Ga}}: \mathrm{As}_{\mathrm{N}}$ ratio changes with the overall Ga:N:As stoichiometry $[13,15]$, so far no direct evidence for so-called $\mathrm{As}_{\mathrm{Ga}}$ "anti-sites" exists, in particular it is unknown whether $\mathrm{As}_{\mathrm{Ga}}$ might be a minority or majority defect in lightly As-doped GaN.

A large number of theoretical studies have investigated the properties of $\mathrm{GaAs}_{1-x} \mathrm{~N}_{x}$ alloys [1, 2, 22-29] and also the nature of As dopants in hexagonal [2, 22-24, 27, 30, 31] and cubic GaN [28, 31-34]. However, most of these studies considered only isoelectronic As atoms substituting for $\mathrm{N}$, thus preserving the cation-anion chemical bonds, and neglecting the possibility of $\mathrm{As}_{\mathrm{Ga}}$ anti-sites. Only Van De Walle and Neugebauer [31] and Ramos et al [34] pointed out that As probably acts as an amphoteric impurity, which may substitute for both $\mathrm{N}$ and Ga.

In this work we have determined the lattice location of radioactive ${ }^{73} \mathrm{As}\left(t_{1 / 2}=80.3 \mathrm{~d}\right)$ in single-crystalline thin films of GaN by means of the emission channeling technique [35] and we present direct experimental evidence that As acts in fact as an amphoteric impurity. Emission channeling is based on the fact that charged particles from nuclear decay $\left(\alpha, \beta^{-}, \beta^{+}\right.$, conversion electrons) experience channeling or blocking effects along major crystallographic axes and planes. The resulting 


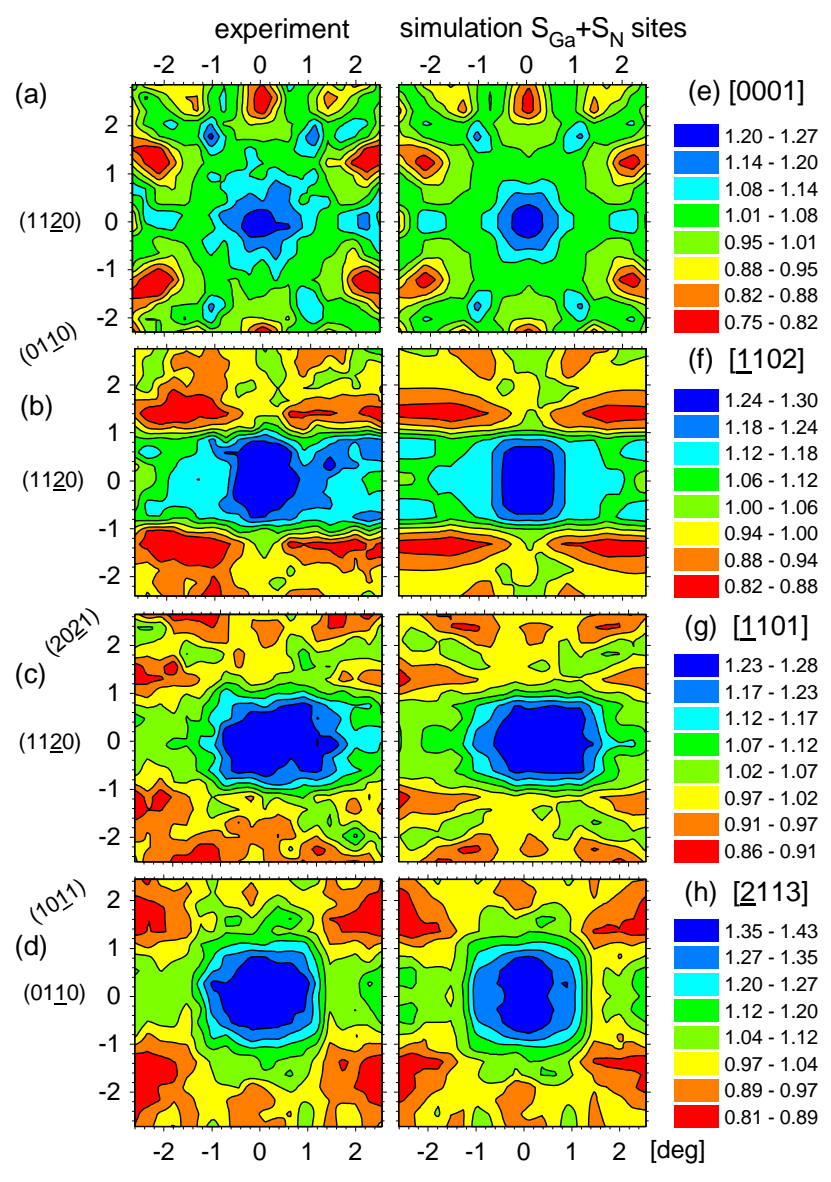

FIG. 1. (Color online) Angular distribution of conversion electron emission yields from ${ }^{73} \mathrm{As} \rightarrow{ }^{73} \mathrm{Ge}$ in GaN following $300^{\circ} \mathrm{C}$ annealing around the (a) [0001], (b) [1102], (c) [1101] and (d) [2113] axis. (e)-(h): best fits of the channeling patterns corresponding to 57(7)\% of probe atoms at substitutional $\mathrm{S}_{\mathrm{Ga}}$ and $48(6) \%$ at $\mathrm{S}_{\mathrm{N}}$ sites.

anisotropic emission yield from the crystal surface characterizes the lattice site occupied by the probe atoms during decay.

The production and ion implantation of ${ }^{73}$ As were performed at CERN's on-line isotope separator facility ISOLDE. The samples were $\sim 1-2 \mu$ m-thick epitaxial wurtzite GaN layers grown on sapphire into which ${ }^{73} \mathrm{As}$ was implanted at room temperature with $60 \mathrm{keV}$ energy at fluences of 0.8 $7 \times 10^{13} \mathrm{~cm}^{-2}$. The As depth profile corresponding to these implantation conditions is centered at a depth of $232 \AA$, with a straggling of $100 \AA$ and the As peak concentration amounting to $0.3-2.7 \times 10^{18}$ atoms $\mathrm{cm}^{-3}$. The maximum As concentration in our experiments was hence still in the $30 \mathrm{ppm}$ regime. The angular emission patterns of the $42.3 \mathrm{keV}$ and $52.1 \mathrm{keV}$ conversion electrons from the ${ }^{73} \mathrm{As} \rightarrow{ }^{73} \mathrm{Ge}$ decay were recorded around the [0001], [1102], [11101] and [2113] directions by means of a position-sensitive Si detector [36]. The evaluation of the probe atom lattice location was performed by quantitatively comparing the experimental patterns with theoretical ones for different lattice sites, using the two-dimensional fit procedure outlined in Ref. [36]. In the fit procedure, we considered theoretical patterns resulting from emitter atoms at substitutional Ga sites $\left(\mathrm{S}_{\mathrm{Ga}}\right)$ and $\mathrm{N}$ sites $\left(\mathrm{S}_{\mathrm{N}}\right)$ with varying root mean square (rms) displacements, the main interstitial sites (cf.

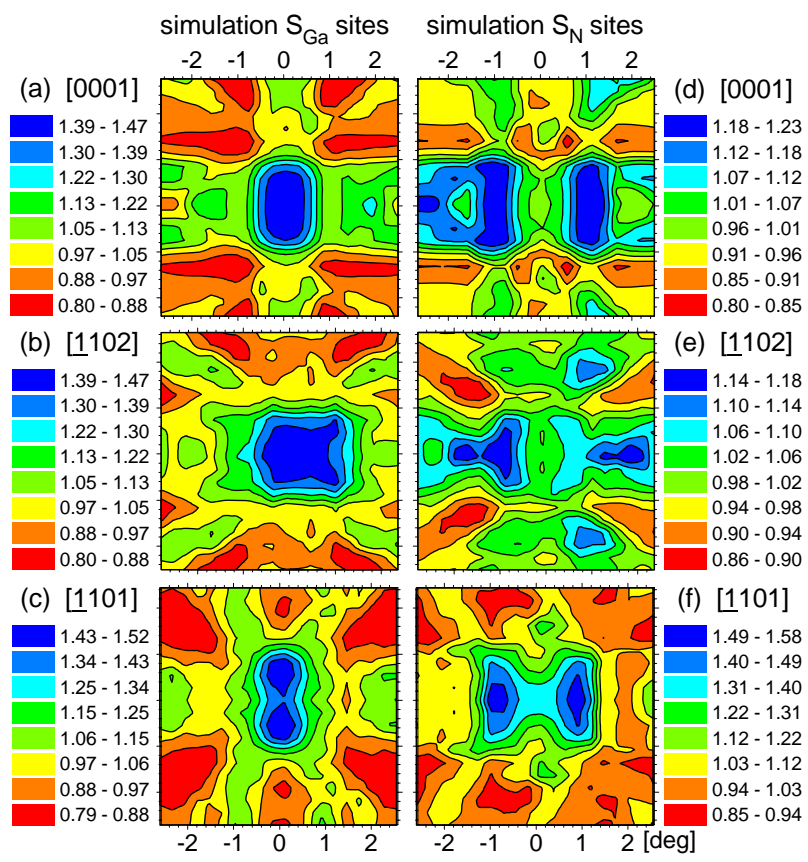

FIG. 2. (Color online) Theoretical emission channeling patterns for substitutional $\mathrm{S}_{\mathrm{Ga}}$ and $\mathrm{S}_{\mathrm{N}}$ sites. (a)-(c): patterns for $100 \%$ of emitter atoms on $\mathrm{S}_{\mathrm{Ga}}$ sites. (d)-(f): patterns for $100 \%$ on $\mathrm{S}_{\mathrm{N}}$ sites.

[37, 38]), and a diversity of interstitial sites resulting from displacements along or off the $c$-axis. The theoretical emission channeling patterns were calculated by means of the "manybeam" theory of electron diffraction in single-crystals [35]. Details with respect to the structural properties of $\mathrm{GaN}$ used in the simulations have been given previously [37].

The experimental emission patterns along the [0001], [1102], [1101] and [2113] directions, following $300^{\circ} \mathrm{C}$ annealing, are shown in Figs. 1 (a)-(d). While the channeling effect along [0001] shows that the emitter atoms are located along the $c$-axis, this does not yet allow distinguishing between $\mathrm{S}_{\mathrm{Ga}}$ and $\mathrm{S}_{\mathrm{N}}$ sites since both are aligned with the [0001] axis. However, it is obvious from a simple visual comparison of the experimental patterns for off-surface directions [Figs. 1 (b)-(d)] to the simulated angular-dependent emission yields for emitter atoms on substitutional $\mathrm{S}_{\mathrm{Ga}}$ and substitutional $\mathrm{S}_{\mathrm{N}}$ sites, which are shown in Fig. 2, that the measured emission channeling effects are dominated by emitter atoms on Ga sites. On the other hand, certain features in the experimental patterns, especially the relatively broad structure of the peaks, cannot be explained by As on Ga sites alone but requires the presence of substantial amounts of As on $\mathrm{N}$ sites. This was fully confirmed by the quantitative results of the fitting procedures. In comparsion to the fit results assuming pure Ga site occupation the chi square of the [1102], [1101] and [2113] fits improved by 39\%, 29\% and $65 \%$ upon introducing a fraction of emitter atoms on substitutional $\mathrm{N}$ sites. The best fits, where the fractions on $\mathrm{S}_{\mathrm{Ga}}$ and $\mathrm{S}_{\mathrm{N}}$ sites and the rms displacement were treated as variable parameters, are shown in Figs. 1 (e)-(h).

Figure 3 shows the derived fractions of emitter atoms on $\mathrm{S}_{\mathrm{Ga}}$ and $\mathrm{S}_{\mathrm{N}}$ sites as a function of annealing temperature. While samples 2, 3 and 4 were only measured in the roomtemperature as-implanted state and sample 5 only after $1000^{\circ} \mathrm{C}$ 


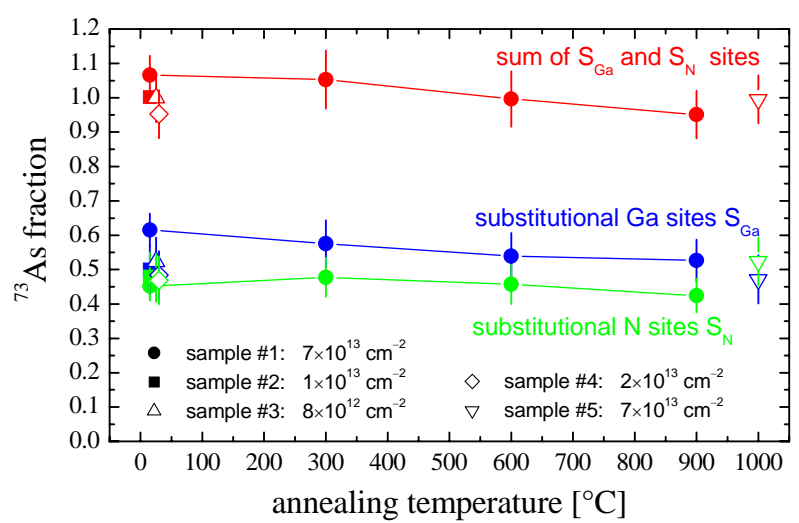

FIG. 3. (Color online) Fractions of probe atoms on substitutional Ga and $\mathrm{N}$ sites and sum of the two fractions as a function of annealing temperature. The implanted fluences of the five samples are indicated.

annealing under nitrogen atmosphere for $30 \mathrm{~min}$, a full annealing sequence (10 min per step under vacuum) was performed for sample 1. In all cases similar fractions of As occupied substitutional Ga and substitutional N sites. The sum fraction in sample 1 is somewhat higher than $100 \%$ but still well within the error of $\pm 10 \%$ introduced by the correction for the background of scattered electrons.

Since various authors [12, 23, 30, 31, 34] have considered the possibility of lattice relaxations related to As in $\mathrm{GaN}$, we would like to comment on this issue. Unfortunately the rms displacements of As from the ideal $\mathrm{S}_{\mathrm{Ga}}$ and $\mathrm{S}_{\mathrm{N}}$ sites could not be unambiguously derived from the fit results, however, both were in the range $0.08-0.19 \AA$, corresponding to $4-10 \%$ of the bond length in pure GaN.

It is interesting to compare the amphoteric nature of As in $\mathrm{GaN}$ with our previous results on the lattice location of implanted As in $\mathrm{ZnO}$ [38], where we found As to prefer substitutional Zn sites with the fraction on $\mathrm{O}$ sites being less than $5 \%$. We attributed this to the large mismatch of the ionic radii of $\mathrm{As}^{3-}(2.22 \AA)$ with $\mathrm{O}^{2-}(1.38 \AA)$ but the good match between $\mathrm{As}^{3+}$ and $\mathrm{Zn}^{2+}(0.58 \AA$ vs $0.60 \AA)$, the electronegativity of As (2.0), which is closer to $\mathrm{Zn}$ (1.6) than to $\mathrm{O}$ (3.5), and its character as a semimetal, all of which make it energetically favorable for the As impurity to be incorporated on Zn sites. In GaN, whereas the ionic radii of $\mathrm{As}^{3+}$ and $\mathrm{Ga}^{3+}$ are also well-matched ( $0.58 \AA$ vs $0.62 \AA$ ), the size-mismatch between $\mathrm{As}^{3-}$ and $\mathrm{N}^{3-}$ ( $2.22 \AA$ vs $1.71 \AA$ ) is considerabaly smaller, which is apparently enough in order to allow for roughly half of it to be incorporated on $\mathrm{N}$ sites.

Summarizing, our findings clearly establish the amphoteric nature of As in GaN. Furthermore, the fact that $\mathrm{As}_{\mathrm{Ga}}$ "antisites" are not minority defects provides an additional factor which has to be taken into account for an explanation of the socalled "miscibility gap" in ternary $\mathrm{GaAs}_{1-x} \mathrm{~N}_{x}$ compounds.

This work was funded by the Portuguese Foundation for Science and Technology (FCT, project POCI-FP-63911-2005) and by the European Commission (EURONS project RII3-CT2004-506065).

\footnotetext{
a) Electronic mail: uwahl@itn.pt

${ }^{1}$ J. Neugebauer and C. Van De Walle, Phys. Rev. B 51, 10568 (1995).
}

2 L. Bellaiche, S.H. Wei, and A. Zunger, Phys. Rev. B 54, 17568 (1996).

${ }^{3}$ W.G. Bi and C.W. Tu, Appl. Phys. Lett. 70, 1608 (1997).

4 Y. Qiu, S.A. Nikishin, H. Temkin, N.N. Faleev, and Y.A. Kudriavtsev, Appl. Phys. Lett. 70, 3242 (1997).

${ }^{5}$ X. Li, E.E. Reuter, S.G. Bishop, and J.J. Coleman, Appl. Phys. Lett. 72, 1990 (1998).

${ }^{6}$ R. Kuroiwa, H. Asahi, K. Asami, S.J. Kim, K. Iwata, and S. Gonda, Appl. Phys. Lett. 73, 2630 (1998).

${ }^{7}$ Y. Zhao, F. Deng, S.S. Lau, and C.W. Tu, J. Vac. Sci. Technol. B 16, 1297 (1998).

${ }^{8}$ H. Na, H.J. Kim, E. Yoon, C. Sone, and Y. Park, J. Cryst. Growth 248, 437 (2003)

${ }^{9}$ J.I. Pankove and J.A. Hutchby, J. Appl. Phys. 47, 5376 (1976).

${ }^{10}$ R.D. Metcalfe, D. Wickenden, and W.C. Clark, J. Lumin. 16, 405 (1978).

${ }^{11}$ A.J. Winser, S.V. Novikov, C.S. Davis, T.S. Cheng, C.T. Foxon, and I. Harrison, Appl. Phys. Lett. 77, 2506 (2000).

${ }^{12}$ B. Gil, A. Morel, T. Taliercio, P. Lefebvre, C.T. Foxon, I. Harrison, A.J.Winser, and S.V. Novikov, Appl. Phys. Lett. 79, 69 (2001).

13 A. Bell, F.A. Ponce, S.V. Novikov, C.T. Foxon, and I. Harrison, Appl. Phys. Lett. 79, 3239 (2001).

${ }^{14}$ H.Y. Huang, J.Q. Xiao, C.S. Ku, H.M. Chung, W.K. Chen, W.H. Chen, M.C. Lee, and H.Y. Lee, J. Appl. Phys. 92, 4129 (2002).

${ }^{15}$ C.T. Foxon, I. Harrison, S.V. Novikov, A.J. Winser, R.P. Campion, and T. Li, J. Phys.: Condens. Matter 14, 3383 (2002).

${ }^{16}$ C.T. Foxon, S.V. Novikov, L.X. Zhao, and I. Harrison, Appl. Phys. Lett. 83, 1166 (2003).

${ }^{17}$ A. Stötzler, R. Weissenborn, M. Deicher, and The ISOLDE collaboration, MRS Internet J. Nitride Semicond. Research 5S1, W12.9 (2000), see also Mater Res. Soc. Symp. Proc. 595, W12.9 (2000).

${ }^{18}$ L.J. Guido, P. Mitev, M. Gherasimova, and B. Gaffey, Appl. Phys. Lett. 72, 2005 (1998).

${ }^{19}$ Y. Tsuda, H. Mouri, T. Yuasa, and M. Taneya, Appl. Phys. Lett. 85, 4361 (2004).

${ }^{20}$ L. Lee, W.C. Lee, H.M. Chung, M.C. Lee, W.H. Chen, W.K. Chen, and H.Y. Lee, Appl. Phys. Lett. 81, 1812 (2002).

${ }^{21}$ M. Ahoujja, S. Elhamri, R. Berney, Y.K. Yeo, and R.L. Hengehold, Mater Res. Soc. Symp. Proc. 831, E3.19 (2005).

${ }^{22}$ L. Bellaiche, S.H. Wei, and A. Zunger, Appl. Phys. Lett. 70, 3558 (1997).

${ }^{23}$ L. Bellaiche, S.H. Wei, and A. Zunger, Phys. Rev. B 56, 10233 (1997).

${ }^{24}$ A. Zunger, phys. stat. sol. (b) 216, 117 (1999).

${ }^{25}$ P.R.C. Kent and A. Zunger, Phys. Rev. B 64, 115208 (2001).

${ }^{26}$ L.W. Wang, Phys. Rev. Lett. 88, 256402 (2002).

${ }^{27}$ J. Li and L.W. Wang, Phys. Rev. B 67, 033102 (2003).

${ }^{28}$ A Jenichen and C. Engler, phys. stat. sol. (b) 241, 1883 (2004).

${ }^{29}$ N. Tit, J. Phys D: Appl. Phys. 39, 2514 (2006).

${ }^{30}$ T. Mattila and A. Zunger, Phys. Rev. B 58, 1367 (1998).

${ }^{31}$ C.G. Van De Walle and J. Neugebauer, Appl. Phys. Lett. 76, 1009 (2000).

32 T. Mattila and A. Zunger, Phys. Rev. B 59, 9943 (1999).

${ }^{33}$ C. Göbel, K. Petzke, C. Schrepel, and U. Scherz, Physica B 273, 759 (1999).

34 L.E. Ramos, J. Furthmüller, J.R. Leite, L.M.R. Scolfaro, and F Bechstedt, Phys. Rev. B 68, 085209 (2003).

${ }^{35}$ H. Hofsäss and G. Lindner, Phys. Rep. 210, 121 (1991).

${ }^{36}$ U. Wahl, J.G. Correia, A. Czermak, S.G. Jahn, P. Jalocha, J.G. Marques, A. Rudge, F. Schopper, J.C. Soares, A. Vantomme, P. Weilhammer, and the ISOLDE collaboration, Nucl. Instr. Meth. A 524, 245 (2004).

${ }^{37}$ U. Wahl, A. Vantomme, G. Langouche, J.P. Araújo, L. Peralta, J.G. Correia, and the ISOLDE collaboration, J. Appl Phys. 88, 1319 (2000).

${ }^{38}$ U. Wahl, E. Rita, J.G. Correia, A.C. Marques, E. Alves, J.C. Soares, and the ISOLDE collaboration, Phys. Rev. Lett. 95, 215503 (2005). 